\title{
Motoneuron Activity Patterns Related to the Earliest Behavior of the Zebrafish Embryo
}

\author{
Louis Saint-Amant and Pierre Drapeau \\ Center for Research in Neuroscience, Montreal General Hospital Research Institute, and Departments of Neurology, \\ Neurosurgery, and Biology, McGill University, Montréal, Québec, Canada H3G 1A4
}

As a first step in the study of the developing motor circuitry of the embryonic zebrafish spinal cord, we obtained patch-clamp recordings in vivo from identified motoneurons in curarized embryos from the onset of the first motor behavior. At an early developmental stage in which embryos showed slow and repetitive spontaneous contractions of the trunk, motoneurons showed periodic depolarizations that triggered rhythmic bursts of action potentials with a frequency and duration that were consistent with those of the spontaneous contractions. The periodic depolarizations were blocked by tetrodotoxin or $\mathrm{Cd}^{2+}$. Surprisingly, the contractions and periodic depolarizations were insensitive to general blockade of synaptic transmission (by elevated $\mathrm{Mg}^{2+}$ and reduced $\mathrm{Ca}^{2+}$, or by $\mathrm{Co}^{2+}$ ) and to selective blockade of the major neurotransmitter receptors of the mature

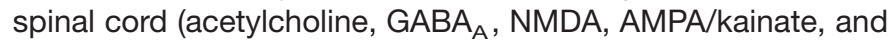
glycine). The periodic depolarizations were suppressed by hep-

An important aspect of basic and clinical neuroscience is understanding the development of the spinal circuits that result in locomotion. It is important to determine the earliest events during synaptogenesis because they likely constitute the backbone for the elaboration of mature circuits and may provide information on fundamental principles of circuit formation common to all vertebrates. The cellular neurophysiology of spinal circuits has been studied in embryonic chick (Chub and O'Donovan, 1998), fetal rat (Ozaki et al., 1996), neonatal rat (Bracci et al., 1996; Cazalets et al., 1996), late embryos of Xenopus (Roberts, 1990), and adult lamprey (Grillner et al., 1991). In all of these species, glutamate was suggested as the major excitatory transmitter, and glycine and GABA were suggested as the major inhibitory transmitters. In addition, a role for electrotonic synapses has been proposed at early stages of neural development (Feller, 1999). However, it remains to be determined how the neural circuits are first established from the beginning of synaptogenesis and how the networks underlying embryonic behaviors are transformed to produce mature locomotion.

The availability of locomotor mutations in the zebrafish embryo (Granato et al., 1996) offers a unique opportunity to analyze spinal cord development at both the cellular and molecular ge-

\footnotetext{
Received Dec. 23, 1999; revised March 6, 2000; accepted March 14, 2000.

This work was supported by a Medical Research Council (MRC) of Canada Studentship to L.S.-A. and by grants from the MRC and Natural Sciences and Engineering Research Council of Canada to P.D. We thank Drs. C. Bourque, P. Carlen, and R. Levine for useful discussions.

Correspondence should be addressed to Dr. Pierre Drapeau, Department of Neurology, Montréal General Hospital, 1650 Cedar Avenue, Montréal, Québec, Canada H3G 1A4. E-mail: mcpd@musica.mcgill.ca.

Copyright (C) 2000 Society for Neuroscience 0270-6474/00/203964-09\$15.00/0
}

tanol or by intracellular acidification, treatments that are known to uncouple gap junctions, indicating that electrotonic synapses could underlie the earliest motor behavior. A few hours later, most motoneurons already showed a new pattern of repetitive activity consisting of bursts of glycinergic synaptic events, but these were not necessary for the spontaneous contractions. Transecting the spinal cord at the hindbrain border did not affect the rhythmic activity patterns of the motoneurons. We suggest that spontaneous contractions of the zebrafish embryo are mediated by an early spinal circuit that is independent of the main neurotransmitter systems and descending hindbrain projections that are required for locomotion in the mature vertebrate spinal cord.

Key words: spinal cord; patch clamp; in vivo; rhythmic activity; synaptic transmission; locomotion netic level, particularly if the architecture of the underlying circuits can be defined. Zebrafish embryos have stereotyped and reproducible developmental stages when raised at $28.5^{\circ} \mathrm{C}(\mathrm{Kim}-$ mel et al., 1995) and show stereotyped motor behaviors (Kimmel et al., 1995; Saint-Amant and Drapeau, 1998). The first motor behavior, which is the focus of this study, consists of side to side contractions of the embryo at $17 \mathrm{hr}$ after fertilization that peak in frequency at $19 \mathrm{hr}$ and decline progressively over the course of $6-7 \mathrm{hr}$.

The spontaneous contractions appear at a time at which few neurons have extended axons in the spinal cord (Bernhardt et al., 1990; Kuwada et al., 1990). These neurons have been classified as seven types of interneurons as well as three primary motoneurons. The first motoneuron to project from the spinal cord is the caudal primary motoneuron $(\mathrm{CaP})$ at $17 \mathrm{hr}$, followed within 1-3 hr by its more rostral counterparts, the middle primary motoneuron $(\mathrm{MiP})$ and the rostral primary motoneuron (RoP). The $\mathrm{CaP}$, MiP, and RoP specifically innervate the ventral, dorsal, and middle muscle mass, respectively, in each somite (Myers et al., 1986). However, it is not known whether the motoneurons are intrinsically active or whether a central pattern generator drives them. As a first step in characterizing the neural basis for the earliest motor behavior, we recorded from motoneurons in vivo from wild-type embryos aged 19-24 hr using recently developed patch-clamp techniques (Drapeau et al., 1999). We did this instead of recording from the minute ventral roots, which at this stage contain only one to three axons that are migrating between muscle fiber layers. We observed two fundamental types of subthreshold activities and describe their development and contributions to the early motor behavior of the zebrafish embryo. 
These results have been published in part in abstract form (Saint-Amant and Drapeau, 1998).

\section{MATERIALS AND METHODS}

Dissections. Embryos were obtained from a zebrafish colony maintained according to established procedures (Westerfield, 1995). Newly fertilized eggs were raised overnight at $26^{\circ} \mathrm{C}$, and once the embryos had 18 somites (corresponding to $18 \mathrm{hr}$ of development) they were incubated until the start of the experiments at $28.5^{\circ} \mathrm{C}$, the standard temperature for staging zebrafish. All procedures were performed in compliance with the guidelines stipulated by the Canadian Council for Animal Care and McGill University. We used zebrafish of the Longfin line. The embryos and larvae were anesthetized and paralyzed, and the muscles overlying two to three somites in the rostral third of the trunk were removed after mild collagenase treatment to expose the spinal cord, as described by Drapeau et al. (1999).

Solutions and recordings. In early experiments $(n=88)$ the recording solution consisted of (in mM): $145 \mathrm{NaCl}, 1.5 \mathrm{KCl}, 2 \mathrm{CaCl}_{2}, 1 \mathrm{MgCl}_{2}, 26$ $\mathrm{NaHCO}_{3}, 1.25 \mathrm{NaH}_{2} \mathrm{PO}_{4}, 10$ glucose, and 0.01 D-tubocurarine, 330 mOsm, $\mathrm{pH} 7.2$, and gassed with $95 \% \mathrm{O}_{2} / 5 \% \mathrm{CO}_{2}$ (Legendre et al., 1994). In later experiments $(n=97)$ we used a recording solution (Drapeau et al., 1999) that was modified from Evans (1979) and consisted of (in mM): $134 \mathrm{NaCl}, 2.9 \mathrm{KCl}, 2.1 \mathrm{CaCl}_{2}, 1.2 \mathrm{MgCl}_{2}, 10$ glucose, 0.01 D-tubocurarine, $290 \mathrm{mOsm}, \mathrm{pH}$ 7.8. Both solutions yielded similar electrophysiological results in embryos with regard to the frequency of events recorded in neurons and thus were pooled for subsequent analysis, but the Evans solution (which lacks $\mathrm{HCO}_{3}{ }^{-}$) gave more consistent chloride reversal potentials and was used particularly for amplitude measurements. The low $\mathrm{Ca}^{2+} /$ high $\mathrm{Mg}^{2+}$ solution consisted of (in mM): 120 $\mathrm{NaCl}, 1 \mathrm{CaCl}_{2}, 10 \mathrm{MgCl}_{2}$, and the other components were the same as the Evans solution described above. In some experiments, tetrodotoxin (TTX; $1 \mu \mathrm{M}$, Sigma, St. Louis, MO), kynurenic acid (2 mM, Sigma), CNQX (5-10 $\mu \mathrm{M}, \mathrm{RBI}$, Natick, MA), APV (40-80 $\mu \mathrm{M}, \mathrm{RBI})$, cobalt (2-4 $\mathrm{mm}$, Sigma), cadmium (100-250 $\mu \mathrm{M}$, Sigma), heptanol (1.5 mM, Sigma), strychnine hydrochloride (1 $\mu \mathrm{M}$, Sigma), $\mathrm{NH}_{4} \mathrm{Cl}$ (20-30 mm, Sigma; added from an isosmotic stock solution),or $\alpha$-bungarotoxin $(10 \mu \mathrm{M}$, Sigma) was added to the superfusion solution.

We used standard whole-cell recording techniques (Hamill et al., 1981) in vivo (Drapeau et al., 1999) at room temperature $\left(22^{\circ} \mathrm{C}\right)$. The recordings usually lasted $\sim 30 \mathrm{~min}$. Patch-clamp electrodes were pulled from thin-walled, Kimax-51 borosilicate glass $(\sim 5 \mathrm{M} \Omega$ resistance) and filled with a potassium gluconate solution consisting of (in mM): 105 potassium gluconate, $16 \mathrm{KCl}, 2 \mathrm{MgCl}_{2}, 10 \mathrm{HEPES}, 10$ EGTA, $4 \mathrm{Na}_{3} \mathrm{ATP}, 273$ mOsm, pH 7.2. The pipette solution had a junction potential of $5 \mathrm{mV}$ that was corrected for. Although active currents could not always be clamped, synaptic currents were effectively space-clamped in these small neurons (Drapeau et al., 1999). In some experiments, the membrane-impermeant sodium channel blocker QX-314 was included in the pipette to block action potentials selectively in the motoneuron recorded from. The lateral surface of the spinal cord was observed under a $40 \times$ waterimmersion objective modified for Hoffman modulation optics. All embryonic motoneurons were labeled during whole-cell experiments for unequivocal identification by including either $0.1 \%$ Lucifer Yellow or sulforhodamineB (Sigma) in the patch pipette, and micrographs were taken of the living preparations before or after removal of the pipette. Whole-cell voltage or current was recorded with an Axopatch-1D amplifier (Axon Instruments), filtered at $2-5 \mathrm{kHz}(-3 \mathrm{~dB})$, and digitized at $20-50 \mathrm{kHz}$. Data were acquired with pClamp 6.0 software (Axon Instruments) and analyzed off-line with Axograph 3.5 and Axoscope software (Axon Instruments). The recordings were not analyzed if the resting potential was more positive than $-40 \mathrm{mV}$ or if the input resistance was below $500 \mathrm{M} \Omega$. Student's $t$ tests were performed to assess the significance between means of paired data.

Behavioral pharmacology. For injections of drugs, dechorionated embryos were immobilized in 1\% low melting point agarose (Sigma) dissolved in embryonic medium (Westerfield, 1995). A saline solution containing Fast Green and the drug of choice was injected into the yolk or forebrain using a Picospritzer (General Valve, Fairfield, NJ). The embryos have no blood circulation before $\sim 23 \mathrm{hr}$ of development; therefore fast green was used to monitor the diff usion of the solution to the trunk of the embryo. The drugs were used at 25-50 times the concentration used for bath application. The embryos were then freed from the agarose, and their motions were recorded on videotape.
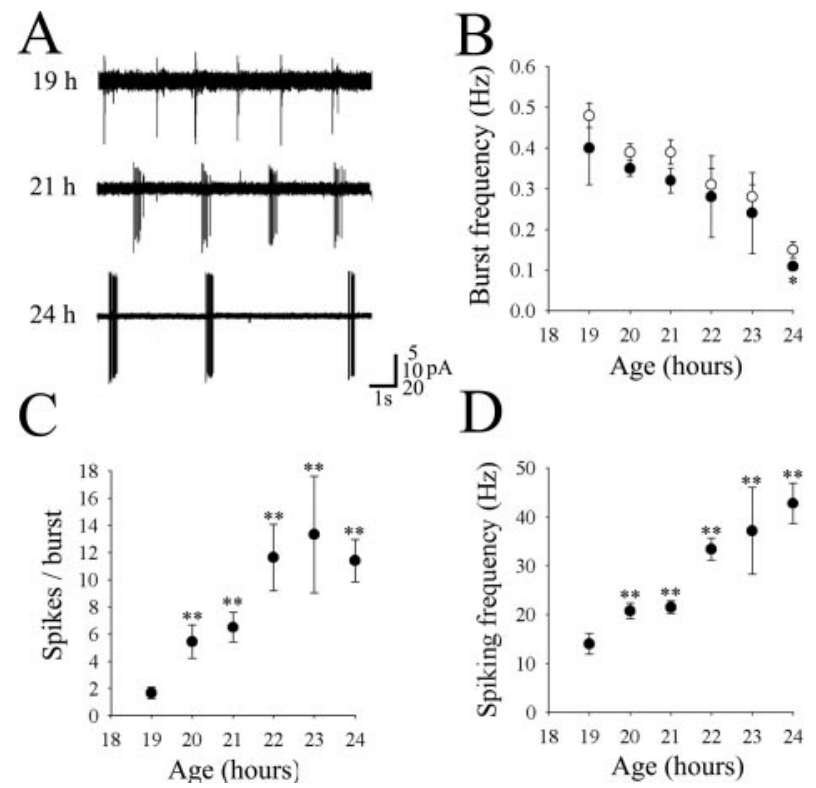

Figure 1. Cell-attached recordings from motoneurons in embryos aged 19-24 hr. A, Examples of recordings at 19, 21, and $24 \mathrm{hr}$. Note the different amplitude scales, and as the embryos matured the bursts contained more spikes. $B$, The average frequency of cell-attached bursts $(\bullet)$ had similar values and showed the same decline as the frequency of contractions in freely moving embryos $(\bigcirc)$ (from Saint-Amant and Drapeau, 1998). $C$, The number of spikes per burst increased dramatically during this short time span of development. $D$, The mean instantaneous frequency of spikes within bursts increased significantly from 19 to $24 \mathrm{hr}$. The asterisks show a significant increase from $19 \mathrm{hr}(p<0.01)$. In this and all other figures, ${ }^{*} p<0.05$ and ${ }^{* *} p<0.01$.

\section{RESULTS}

\section{Cell-attached recordings}

Embryos were paralyzed with either D-tubocurarine or $\alpha$-bungarotoxin to permit neuronal recordings. Neither agent appeared to affect cell firing patterns or synaptic activity (see below) in the embryos, which was also observed during preliminary recordings from larval motoneurons (Buss et al., 1999). When recording extracellularly from motoneurons in the cellattached configuration, no activity was seen until $19 \mathrm{hr}$, when repetitive bursts of (extracellular) action potentials were first observed (Fig. 1A). Although these extracellular recordings provide less information, they are less invasive than whole-cell recordings and could therefore reflect more accurately the motoneuron firing patterns in the intact animal. Recordings were obtained from 25 different motoneurons in embryos aged 19-24 hr. The bursts changed considerably in appearance and frequency during this brief, $5 \mathrm{hr}$ time interval. This is illustrated in Figure $1 A$, which shows sample recordings obtained at different times in development. The bursts occurred with a peak frequency of $0.40 \pm 0.09 \mathrm{~Hz}$ at $19 \mathrm{hr}$ and then decreased significantly to $0.11 \pm$ $0.01 \mathrm{~Hz}$ at $24 \mathrm{hr}($ Fig. $1 B, \bigcirc)(p<0.05)$. These values were not significantly different from the measurements obtained in our previous behavioral study of contraction cycle frequencies for each time point, which suggests that each burst could underlie one of the slow coiling contractions seen in the embryos. (Fig. $1 B, \bigcirc$; from Fig. 2 of Saint-Amant and Drapeau, 1998). The number of action potentials in each burst increased rapidly from $1.7 \pm 0.4$ spikes at $19 \mathrm{hr}$ to $11 \pm 2$ spikes at $24 \mathrm{hr}$ (Fig. 1C). The mean duration of the bursts from 20 to $24 \mathrm{hr}$ was $340 \pm 19 \mathrm{msec}(n=$ 21 cells). No variation in the duration of bursting attributable to 

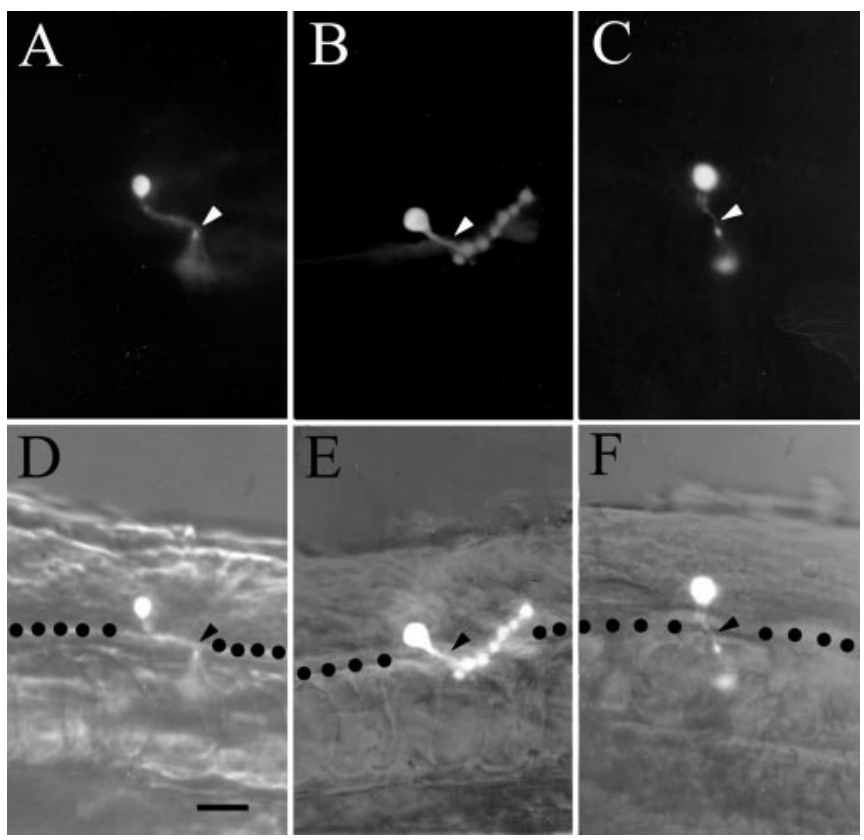

Figure 2. Examples of the three types of motoneurons recorded from. $A-C$, Fluorescence images (Lucifer yellow). In all cases the axons reached out of the plane of focus to project between the two overlying muscle layers. $D-F$, The same cells photographed in bright-field with Hoffman modulation optics. $A, D$, A RoP motoneuron at $24 \mathrm{hr}$. The axon projected caudally (to the right) from the cell body toward the ventral border of the spinal cord (dotted line) and then projected out of the spinal cord via the ventral root to the midline muscle mass. $B, E, \mathrm{~A} \mathrm{MiP}$ motoneuron at $22 \mathrm{hr}$. The axon projected caudally, exited the ventral root, and projected to the dorsal muscle mass. $C, F, \mathrm{~A} \mathrm{CaP}$ at $20 \mathrm{hr}$. The axon projected directly to the ventral root, exited, and headed for the ventral muscle. Scale bar, $25 \mu \mathrm{m}$.

maturation was detected, and no significant difference in burst duration was observed between any of the age groups. As the number of spikes per burst increased with age but the burst duration remained constant, the spiking frequency within the bursts increased progressively from $14 \pm 2 \mathrm{~Hz}$ at $19 \mathrm{hr}$ to $43 \pm 4$ $\mathrm{Hz}$ at $24 \mathrm{hr}(p<0.01)$ (Fig. 1D). These sharp increases in extracellular spike frequency and amplitude (Fig. $1 A$ ) presumably reflect the addition of sodium channels in the maturing motoneurons.

\section{Whole-cell recordings from motoneurons}

The patch of membrane under the pipette was ruptured to pass to the whole-cell recording configuration in 61 cells. Lucifer Yellow or sulforhodamineB was included in the pipette for identification of all cells. At the earliest stages $(<19 \mathrm{hr})$, cells that were obtained lacked axons, action potentials, synaptic activity, and rhythmic activity (data not shown). Motoneurons were identified on the basis of their size, ventral location, and axonal projections to the musculature (Myers et al., 1986). Figure 2 shows a RoP at $20 \mathrm{hr}(A, D)$, a $\mathrm{MiP}$ at $22 \mathrm{hr}(B, E)$, and a $\mathrm{CaP}$ at $20 \mathrm{hr}(C, F)$. Motoneurons with axons that exited the ventral roots showed action potentials and also displayed rhythmic subthreshold activities, as described below. Two types of spontaneous activity patterns were observed, and the detailed justification for naming each type will be provided below. Because all motoneurons showed similar activity patterns, we have grouped the data together. This activity was highly regular, did not contain long pauses or periods of hyperactivity, and persisted throughout the recording period.

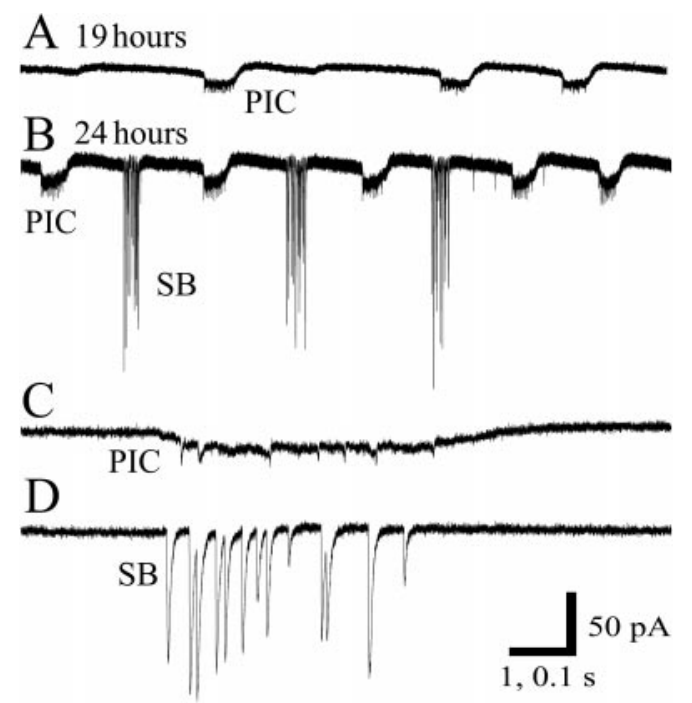

$\mathrm{E}$

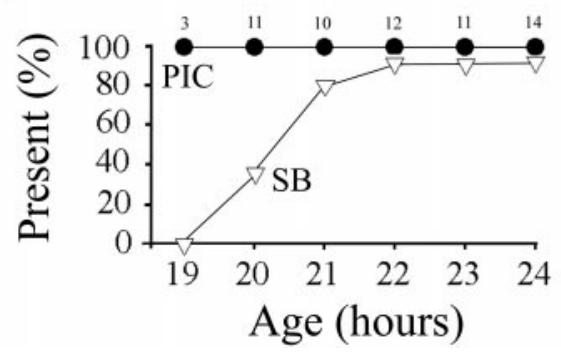

Figure 3. Whole-cell voltage-clamp recordings of motoneurons at 19 and $24 \mathrm{hr}$. $A$, Recording at $19 \mathrm{hr}$ showing only periodic inward currents $(P I C)$. $B$, A recording at $24 \mathrm{hr}$ showing both PIC and synaptic bursts $(S B)$. $C, D$, Segments from the recording in $B$ are shown on an expanded time scale. PICs were composed of a slow and low amplitude inward current followed by a smaller, transient outward current (seen more clearly in $A$ and $B$ ). SBs were composed of synaptic currents of large amplitude, and each event had a rapid time course. $E$, Age of onset of each activity pattern. The graphs display the percentage of the cells expressing each type of activity versus the age at which the recording was made. PICs $(\bullet)$ were present in all of the earliest recordings. SBs ( $\nabla)$ appeared later than the waves but were nevertheless present in most of the cells by $21 \mathrm{hr}$. The numbers above each point indicate the number of cells recorded from for each age group.

The first type of activity observed, under voltage clamp, was a periodic inward current (Fig. $3 A, C, P I C$ ) consisting of low amplitude $(<20 \mathrm{pA})$ inward current steps of long duration (400-600 msec) followed by smaller, transient outward currents. Small spikelets were usually seen during the periodic inward current. The second type of activity observed was a burst of synaptic events, or synaptic burst (Fig. $3 B, D, S B$ ), in which each synaptic event within a burst was larger in amplitude and faster in time course than the episodes of periodic inward current. However, the total duration and frequency of the synaptic bursts were comparable to the periodic inward currents. When recorded in currentclamp mode, the periodic inward currents gave rise to periodic depolarizations, whereas the bursts of synaptic currents led to bursts of synaptic potentials. Quantitative measurements, such as amplitude and duration, were always performed in voltage clamp because the two activity patterns were easier to distinguish and the currents (being independent of input resistance) were a more reliable source of data for comparisons.

The periodic inward currents were present in all of the record- 

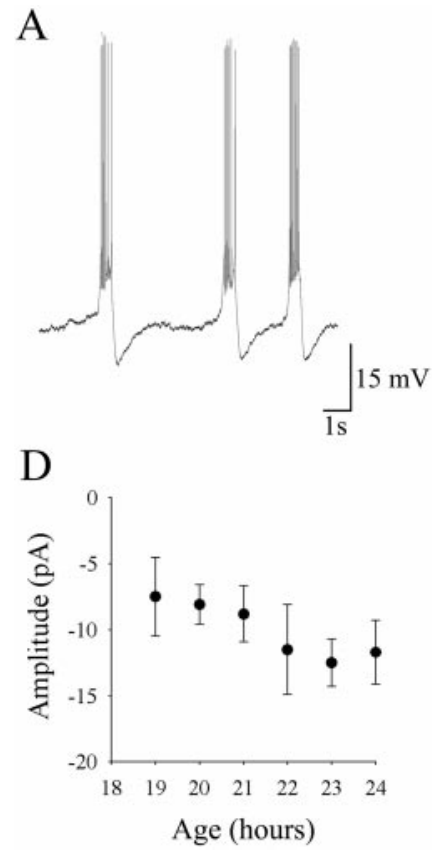

B

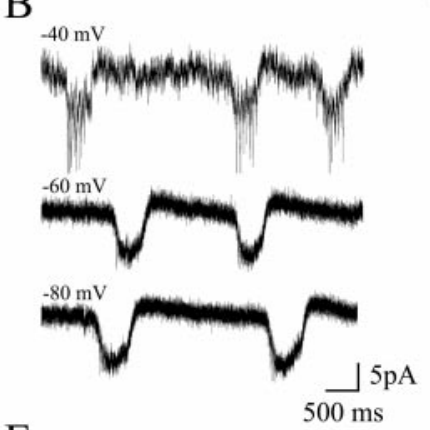

E

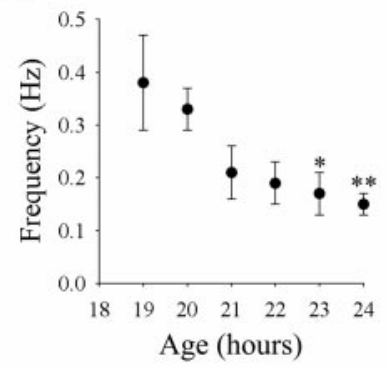

C

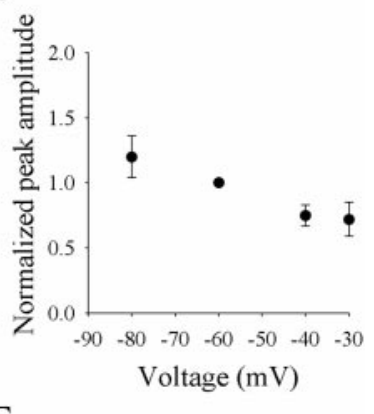

$\mathrm{F}$

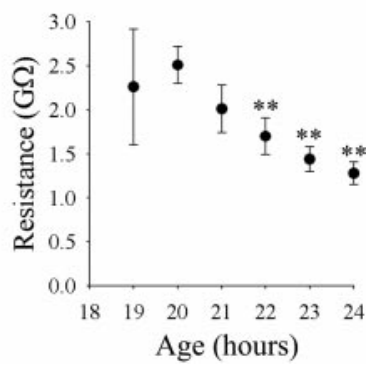

Figure 4. Properties and maturation of periodic inward currents. $A$, Current-clamp recording from a motoneuron at $20 \mathrm{hr}$ showing only periodic depolarizations. The baseline was at $-55 \mathrm{mV}$, and spikes were generated during the episodes of periodic depolarizations. B, Voltage-clamp recordings from a $20 \mathrm{hr}$ motoneuron at different holding potentials. $C$, Normalized amplitude versus membrane voltage $(n=7$ cells). The amplitude of episodes of periodic inward currents changed little over a substantial voltage range. $D$, The amplitude of the periodic inward currents (at a holding potential of $-60 \mathrm{mV}$ ) did not change significantly from 19 to $24 \mathrm{hr}$ and was approximately $-10 \mathrm{pA}$. E, Average frequency of periodic inward currents sampled at different ages. As for the behavior, there was a gradual decrease in the average frequency over time. $F$, The input resistance of the motoneurons decreased significantly from 20 to $24 \mathrm{hr}$. ings from the earliest time obtained (19 hr); the synaptic bursts appeared only in a low percentage of cells at $20 \mathrm{hr}$ and then gradually increased in occurrence over the course of $2 \mathrm{hr}$ (Fig. $3 E$ ). There appeared to be some degree of alternation between synaptic bursts and periodic inward currents. To estimate the degree of alternation, we examined each event in 5 min recordings from each of three embryos at different ages. A value of 0 was assigned if an event was followed by one of the same type, or a value of 1 was assigned if an event was followed by a different type of event. Thus, an overall value of 0.5 would indicate random alternation between periodic inward currents and synaptic bursts. We found an overall value of 0.7 at $20-22 \mathrm{hr}$ and 0.5 at $23-24 \mathrm{hr}$, indicating that alternating activity patterns were more likely in young embryos but later became more randomized because of misalternation. After $23 \mathrm{hr}$, uncommon events $(<5 \%)$ appeared to be mixed bursts consisting of overlapping episodes of periodic inward currents and synaptic bursts (data not shown). We describe the features of each of the two major activity patterns in turn below.

\section{Periodic depolarizations}

In current-clamp recordings (Fig. $4 A$ ) the periodic depolarizations gave rise to a burst of spikes, followed by an afterhyperpolarization. In this example from a young $(20 \mathrm{hr})$ embryo, only periodic depolarizations were detected. When in voltage clamp, the same cell showed periodic inward currents (Fig. 4B). Periodic depolarizations (or periodic inward currents in voltage clamp) were seen in all of the whole-cell recordings $(n=61)$ at all ages. As with the duration of the cell-attached bursts, the duration of periodic inward currents did not vary significantly from $19 \mathrm{hr}$ $(468 \pm 17 \mathrm{msec})$ to $24 \mathrm{hr}(417 \pm 61 \mathrm{msec})$. Periodic inward currents had average peak amplitudes of $-10 \pm 1 \mathrm{pA}(n=27$ cells) at a holding potential of $-60 \mathrm{mV}$. Although there was a trend toward smaller peak amplitudes at more depolarized holding potentials, this decrease was not significant from -60 to -30 $\mathrm{mV}$ (Fig. 4B,C). Reversal of the events was attempted with $4 \mathrm{mM}$ QX-314 in the pipette to block action potentials, but the baseline noise increased at membrane potentials positive to $-20 \mathrm{mV}$ and consequently the periodic inward currents became undetectable. Although we did not observe a significant dependence of the current amplitude on holding potential, variations in membrane voltage did have an effect on the frequency of the periodic inward currents. Stepping the membrane voltage from $-60 \mathrm{mV}$ to -40 $\mathrm{mV}$ resulted in a significant increase in instantaneous frequency of $61 \%(n=11, p<0.001)$. Conversely, changing the membrane potential from $-60 \mathrm{mV}$ to $-80 \mathrm{mV}$ resulted in a significant decrease in frequency of $32 \%(n=11, p<0.01)$.

The peak amplitude at $-60 \mathrm{mV}$ did not change significantly with development from $19 \mathrm{hr}(-8 \pm 3 \mathrm{pA})$ to $24 \mathrm{hr}(-12 \pm 2 \mathrm{pA})$ (Fig. 4D). Like the behavioral frequency of contractions, the frequency of periodic inward currents decreased with development, with a peak at $0.37 \pm 0.09 \mathrm{~Hz}$ at $19 \mathrm{hr}$ that decreased to $0.14 \pm 0.02 \mathrm{~Hz}$ at $24 \mathrm{hr}(p<0.01)$ (Fig. $4 E)$. As expected, the frequencies of periodic depolarizations at each age were not significantly different from the spike burst frequencies observed in cell-attached recordings. Periodic depolarizations were the only activity patterns observed at $19 \mathrm{hr}$ that generated spiking activity in the motoneurons, suggesting that they alone could underlie the spike bursts seen in the motoneurons and consequently the behavioral contractions. The input resistance of the cells also decreased with development, with the peak resistance dropping from $2.5 \pm 0.2 \mathrm{G} \Omega$ at $20 \mathrm{hr}$ to $1.2 \pm 0.1 \mathrm{G} \Omega$ by $24 \mathrm{hr}$ (Fig. $4 F$ ) $(p<0.05)$ and reaching $0.49 \pm 0.06 \mathrm{G} \Omega$ by $2-3 \mathrm{~d}$ (Drapeau et al., 1999).

\section{Synaptic bursts}

Synaptic bursts appeared later than periodic depolarizations, never triggered spikes in current-clamp recordings (Fig. 5A), and consisted of large and fast individual current events in voltageclamp recordings (Fig. 5B). From 20 to $24 \mathrm{hr}$, the frequency of synaptic bursting was not significantly different from that of the periodic inward currents, and like the periodic inward currents the duration of synaptic bursts did not change significantly with development, with an average of $378 \pm 28 \mathrm{msec}(n=17)$. In contrast to the periodic inward currents, the mean peak amplitude of synaptic bursts (estimated as the average of 10-20 events 
A

Figure 5. Properties and maturation of synaptic bursts. $A$, Current-clamp recording from a motoneuron at $24 \mathrm{hr}$. $B$, Recordings from a $22 \mathrm{hr}$ motoneuron at different holding potentials. $C$, Normalized amplitude versus membrane potential ( $n=8$ cells). The mean amplitude of synaptic potentials decreased with increasing voltage and reversed near $-40 \mathrm{mV}$. D, The amplitude of synaptic events was low ( $<20 \mathrm{pA}$ ) at 20 and $21 \mathrm{hr}$ but increased dramatically by $22 \mathrm{hr}$. $E$ shows that the mean instantaneous frequency increased dramatically from $11 \mathrm{~Hz}$ at $20 \mathrm{hr}$ to $51 \mathrm{~Hz} . F$, In some cell-attached recordings, synaptic bursts could be detected (SB, top trace) and were found to result from a current with the same polarity as the sodium current during action potential bursts $(A P B$, bottom trace; note the different scale).

D
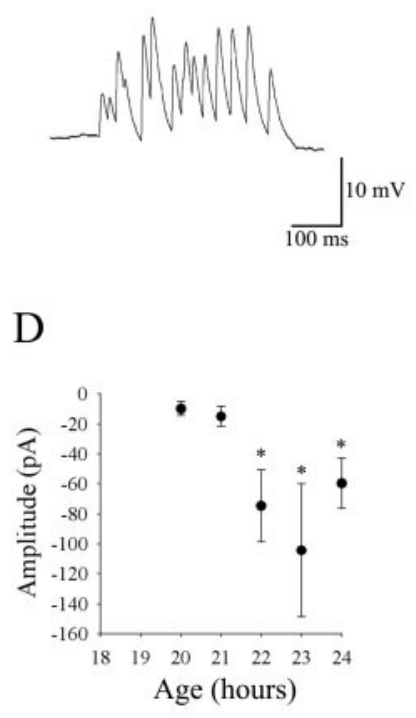

B

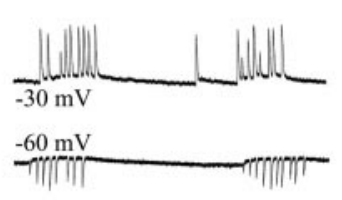

C

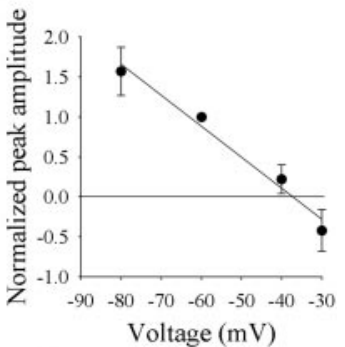

$\mathrm{E}$
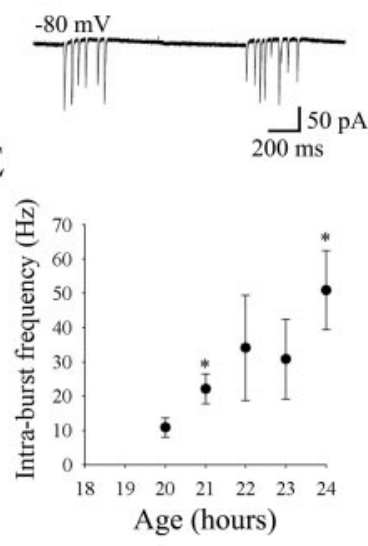
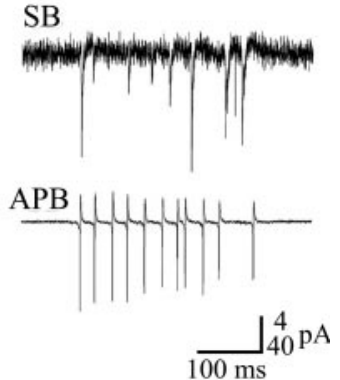

detected in several consecutive bursts) was markedly affected by changes in the holding potential of the cells (Fig. 5B,C). Events decreased in size when the cells were depolarized and reversed polarity at $-38 \pm 4 \mathrm{mV}(n=8)$ (Fig. $5 C)$. Because the estimated reversal potential for chloride is $-49 \mathrm{mV}$, assuming complete dialysis of the cells with the intracellular solution, this suggests that the synaptic events are caused mainly by increases in chloride conductance. During development, the mean peak amplitude of synaptic events recorded at $-60 \mathrm{mV}$ increased from $-10 \pm 3 \mathrm{pA}$ at $20 \mathrm{hr}$ to values greater than $-60 \mathrm{pA}$ by $22 \mathrm{hr}$ (Fig. $5 D$ ). The amplitude values obtained after $21 \mathrm{hr}$ were variable, however, and were not significantly different from each other but were significantly larger than the values at 20 and $21 \mathrm{hr}$. Three of the 11 cells recorded at $20 \mathrm{hr}$, the earliest time of occurrence of synaptic bursts, showed no events at $-60 \mathrm{mV}$, but hyperpolarizing them to $-100 \mathrm{mV}$ unmasked synaptic bursts (data not shown). This suggests that the events were present in some cells at $20 \mathrm{hr}$ but were of such low amplitude at the resting potential that the currents at these presumably immature synapses were sometimes lost in the noise. Interestingly, the intraburst synaptic event frequency was similar to the cell-attached spiking frequencies, increasing from $11 \pm 3 \mathrm{~Hz}$ at $20 \mathrm{hr}$ to $51 \pm 12 \mathrm{~Hz}$ at $24 \mathrm{hr}$ (Fig. $5 E$ ).

The synaptic events were caused by inward currents under resting conditions, as detected in cell-attached (extracellular) recordings of capacitive currents conducted through the patch of membrane under the electrode seal. In these recordings, the events had polarities similar to the initial current during an action potential (Fig. $5 F$ ). This suggests that the intracellular chloride concentration is normally high, as is typically the case in neurons of vertebrate embryos (Cherubini et al., 1991; Singer et al., 1998). It is interesting to note that the instantaneous frequency of the synaptic bursts showed responses to changes in membrane potential, which were the opposite of what was seen with periodic inward currents. Stepping the membrane potential from $-60 \mathrm{mV}$ to $-40 \mathrm{mV}$ resulted in a small but significant decrease in frequency of $19 \%(n=11, p=0.008)$. In contrast, changing the membrane potential from $-60 \mathrm{mV}$ to $-80 \mathrm{mV}$ resulted in an increase in frequency of $18 \%(n=11, p=0.03)$.

\section{Effects of spinalization on activity patterns}

We showed previously (Saint-Amant and Drapeau, 1998) that spinalization did not affect the spontaneous contractions. In this study, complete isolation of the spinal cord from the hindbrain failed to affect the periodic inward currents and synaptic bursts as measured by their durations, amplitudes, and frequencies $(n=8)$. These results indicate that a local, spinal circuit mediates the periodic depolarizations and synaptic bursts.

\section{Pharmacology of activity patterns}

Various specific antagonists of the major transmitters of the mature vertebrate spinal cord were used to assess the nature of the transmitters mediating the different activity patterns. As mentioned above, D-tubocurarine and $\alpha$-bungarotoxin had no noticeable effects, indicating that cholinergic inputs were probably not participating in the generation of periodic depolarizations. Glutamatergic blockers (2 mm kynurenic acid, $40 \mu \mathrm{M}$ APV, $10 \mu \mathrm{M}$ CNQX) did not block the periodic inward currents $(n=10)$ or the synaptic bursts $(n=6)$ (Fig. $6 A)$. Several lines of evidence suggest that these blockers are effective at blocking glutamate receptors in zebrafish. First, the same concentrations of blockers eliminated spontaneous miniature events as well as touch-evoked contractions that were observed in embryos aged 22-24 hr (our unpublished observation). Second, these blockers eliminated miniature EPSCs in older larvae (Ali et al., 2000). Finally, these compounds were also shown to block rhythmic activity in motoneurons of zebrafish larvae (Drapeau et al., 1999). Taken together, these results indicate that an effective block of both spontaneous and evoked chemical synaptic activity by CNQX, APV, and kynurenic acid did not prevent the generation of periodic depolarizations in the early embryos.

To test whether inhibition could support rhythmic activity, we examined the effects of glycinergic and GABAergic antagonists. Addition of strychnine $(1 \mu \mathrm{M})$ to the bath did not affect the amplitude of periodic inward currents $(n=10)$ but resulted in the almost complete (and reversible) loss of synaptic bursts (Fig. 6B). These results indicate that the bursts of synaptic events are glycinergic, whereas neither glycine nor glutamate mediates the periodic inward currents. In addition to the periodic inward 
A

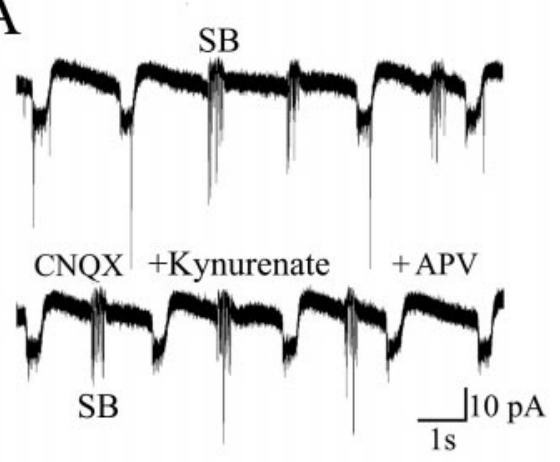

C
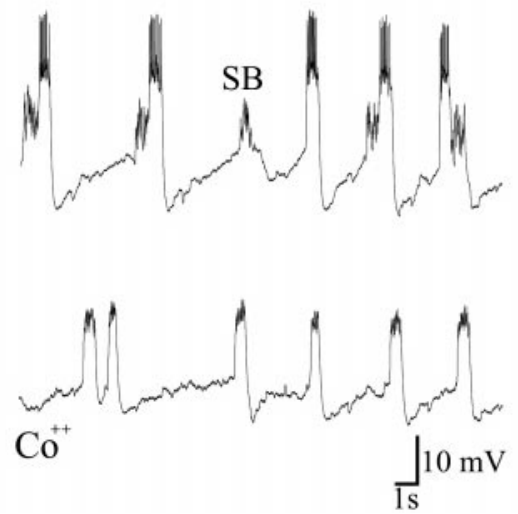

B
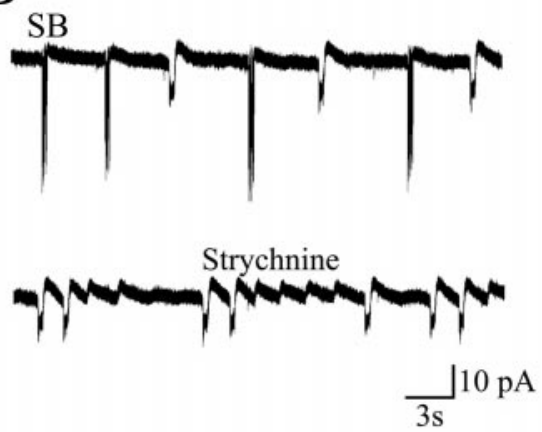

D
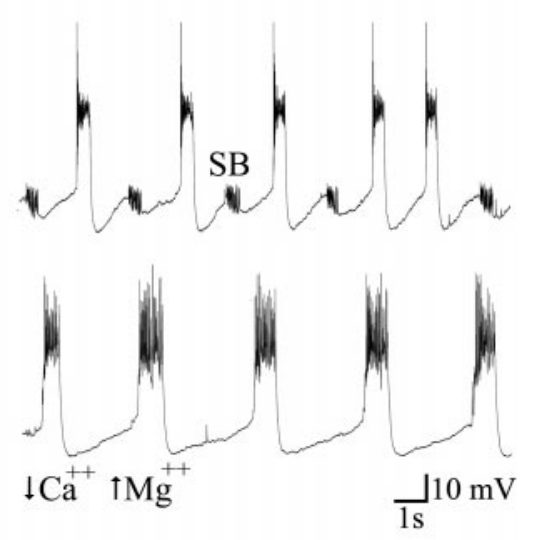

Figure 6. Pharmacology of activity patterns. A, CNQX, kynurenate, and APV (5 min) had no effect on the rhythmic activity. $B$, Strychnine blocked the synaptic bursts but not the periodic inward currents. $\mathrm{C}$, Cobalt $\left(\mathrm{Co}^{++}, 2 \mathrm{mM}\right)$ blocked synaptic bursts but not periodic depolarizations. $D$, Similarly, the low $\mathrm{Ca}^{2+} /$ high $\mathrm{Mg}^{2+}$ solution blocked synaptic bursts but not periodic depolarizations. currents, small, residual currents were sometimes observed in the presence of strychnine (Fig. 6B), suggesting that they may have coincided with the synaptic bursts. Similar small events were observed in some cells, even in the absence of strychnine, when a small inward current occurred during the burst of synaptic activity (Fig. 5B). (Their possible source will be considered in Discussion.) Addition of both glycinergic and glutamatergic antagonists was also without effect on the periodic inward currents or the small, residual events $(n=9)$. The effects of bicuculline were next examined to assess whether GABA receptors played a role in the generation of periodic inward currents. Bicuculline $(25 \mu \mathrm{M}) \mathrm{did}$ not block periodic inward currents and synaptic bursts but, particularly in older embryos, increased significantly the duration of both types of events (e.g., from $376 \pm 17$ to $627 \pm 72$ msec at 23 hr; $n=5)$. Together these observations indicate that none of the ubiquitous neurotransmitters of the mature spinal cord (acetylcholine, GABA, glutamate, and glycine) play a significant role in initiating the periodic inward currents underlying the early contractions of the embryo.

To test for the possible role of another, unusual type of chemical transmitter released in the embryonic spinal cord and to test for intrinsic mechanisms in the motoneurons, we applied various general blockers of synaptic transmission. Calcium channels are generally implicated in the generation of depolarizations such as plateau potentials and in the intrinsic pacemaker abilities of some cells and are crucial for chemical synaptic transmission (Katz and Miledi, 1967; Kiehn, 1991). Cadmium $\left(\mathrm{Cd}^{2+}\right)$ and cobalt $\left(\mathrm{Co}^{2+}\right)$ are divalent cations that can nonspecifically block many types of calcium channels. Adding $200 \mu \mathrm{M} \mathrm{Cd}^{2+}$ in the bath blocked all rhythmic activity within $60 \mathrm{sec}$ of application $(n=5)$. On washout, periodic inward currents returned before the synaptic bursts reappeared. However, in the presence of $2 \mathrm{mM} \mathrm{Co}^{2+}$ (Fig. $6 C$ ), synaptic bursts were reversibly eliminated, whereas periodic in- ward currents remained $(n=4)$. When applied to the bath, the sodium channel blocker TTX ( $1 \mu \mathrm{M}$; data not shown) inhibited all activity at all of the embryonic stages examined, including periodic inward currents $(n=13)$ and synaptic bursts $(n=11)$. Blocking action potentials selectively in the cell recorded from, by including impermeant QX-314 (4 mm; data not shown) in the pipette, eliminated spikes in that cell but had no effect on any of the motoneuron activity patterns $(n=4)$. A similar block of ventral root activity in fetal rat by TTX and $\mathrm{Cd}^{2+}$ has been observed (Ozaki et al., 1996). The high sensitivity to TTX but not to QX-314 points to a need for spiking activity in a premotoneuronal network and also to a lack of cell-intrinsic oscillatory mechanisms that are independent of voltage-sensitive sodium channels. Finally, a solution containing a low concentration of $\mathrm{Ca}^{2+}$ and a high concentration of $\mathrm{Mg}^{2+}$ was used. The low $\mathrm{Ca}^{2+} /$ high $\mathrm{Mg}^{2+}$ solution significantly reduced the amplitude and occurrence of synaptic bursts $(n=7)$ but did not block the periodic inward currents $(n=14)$ (Fig. $6 D)$, although it caused a significant increase in the duration of periodic inward currents. None of these treatments noticeably affected the resting potential. It is concluded from these experiments that although chemical synapses underlie synaptic bursts and can modify some parameters of periodic inward currents, they do not seem to be involved in the generation of periodic inward currents. Because the periodic inward currents were insensitive to $\mathrm{Co}^{2+}$ (which nonetheless blocked synaptic bursts), a calcium conductance different from the one needed for synaptic transmission may contribute to the premotor network activity generating the periodic inward currents.

Because chemical synaptic transmission did not seem to be responsible for the periodic inward currents, the possible role for electrical coupling between premotoneurons and motoneurons during this activity leading to spontaneous contractions was 
tested. In a small fraction (3/61) of the recordings, we observed labeling of another neuron presumably coupled to the motoneuron that was recorded from (data not shown), but these incidences of coupling were too few to be confident about the presence of gap junctions. As a more compelling test of whether gap junctions could play a role in the generation of the periodic inward currents, the preparation was treated with heptanol, which blocks gap junctions when applied to the bath at concentrations ranging from 1 to $10 \mathrm{~mm}$ (Takens-Kwak et al., 1992). Heptanol (2 mm) blocked all rhythmic activity $(n=6)$. In all experiments, action potentials could still be elicited in the presence of heptanol (data not shown), and rhythmic activity recovered on washout of the heptanol, suggesting a benign effect of heptanol on cell physiology, although other side effects cannot be ruled out. As an independent test for gap junctions, we attempted to lower the internal $\mathrm{pH}$ because this is known to block gap junctional coupling (Spray et al., 1981). An ammonia rebound protocol was used to change the intracellular $\mathrm{pH}$ (Roos and Boron, 1981; Nachshen and Drapeau, 1988). This consisted of washing in $\mathrm{NH}_{4} \mathrm{Cl}$ to produce an alkalization of the cytoplasm caused by diffusion of permeant $\mathrm{NH}_{3}$, which can then associate with intracellular $\mathrm{H}^{+}$ions; washing out the $\mathrm{NH}_{4} \mathrm{Cl}$ then produces the reverse, a transient acidification until all the $\mathrm{NH}_{3}$ has left the cell. After a 3 min control recording, a concentration of $20-30 \mathrm{mM} \mathrm{NH}_{4} \mathrm{Cl}$ was washed into the bath for $6 \mathrm{~min}$ and then washed out for $15 \mathrm{~min}$ (Fig. 7A). During the wash-in (and presumed alkalization), the cells were depolarized and the events were of longer duration and larger amplitude, but the average frequency of periodic depolarization remained constant $(n=6)$ (Fig. $7 A-C)$. During the wash-out (and presumed rebound acidification), the bursting activity nearly ceased; there was a significant reduction in the average frequency of periodic depolarizations from $0.26 \pm 0.04 \mathrm{~Hz}$ in the control to $0.06 \pm 0.02$ $\mathrm{Hz}$ during the first $6 \mathrm{~min}$ of washout $(n=6)$ (Fig. $7 A-C)$. Although neither the heptanol nor the $\mathrm{NH}_{4} \mathrm{Cl}$ experiments alone are conclusive, taken together they are consistent with a role for gap junctional coupling between premotoneurons and motoneurons.

To correlate the motoneuron activity patterns with the motor behavior, we examined the effect of various pharmacological manipulations on the spontaneous contractions in the freely behaving animal. Strychnine injection or bath application did not affect the spontaneous contractions $(n=16)$. Likewise, injection of both APV and CNQX had no effect on the spontaneous contractions $(n=31)$. Finally, in the presence of $2 \mathrm{~mm}$ heptanol, the embryos $(n=16)$ were completely immotile and recovered on washout of heptanol, but an effect on electrical coupling between the muscle cells (Nguyen et al., 1999) may have contributed to the suppression of the trunk contractions. These results show that neither glutamatergic nor glycinergic transmission was required for spontaneous contractions or for their electrophysiological correlate, the periodic inward currents, and that these may require electrical connections instead.

\section{DISCUSSION}

\section{Spontaneous contractions and periodic depolarizations}

Spontaneous contractions have been known to occur in embryos of various fishes and were thought to be myogenic in dogfish (Harris and Whitting, 1954). Observations in zebrafish have shown that the neuromuscular synapse blockers D-tubocurare and $\alpha$-bungarotoxin completely block spontaneous contractions (Grunwald et al., 1988; Saint-Amant and Drapeau 1998), which
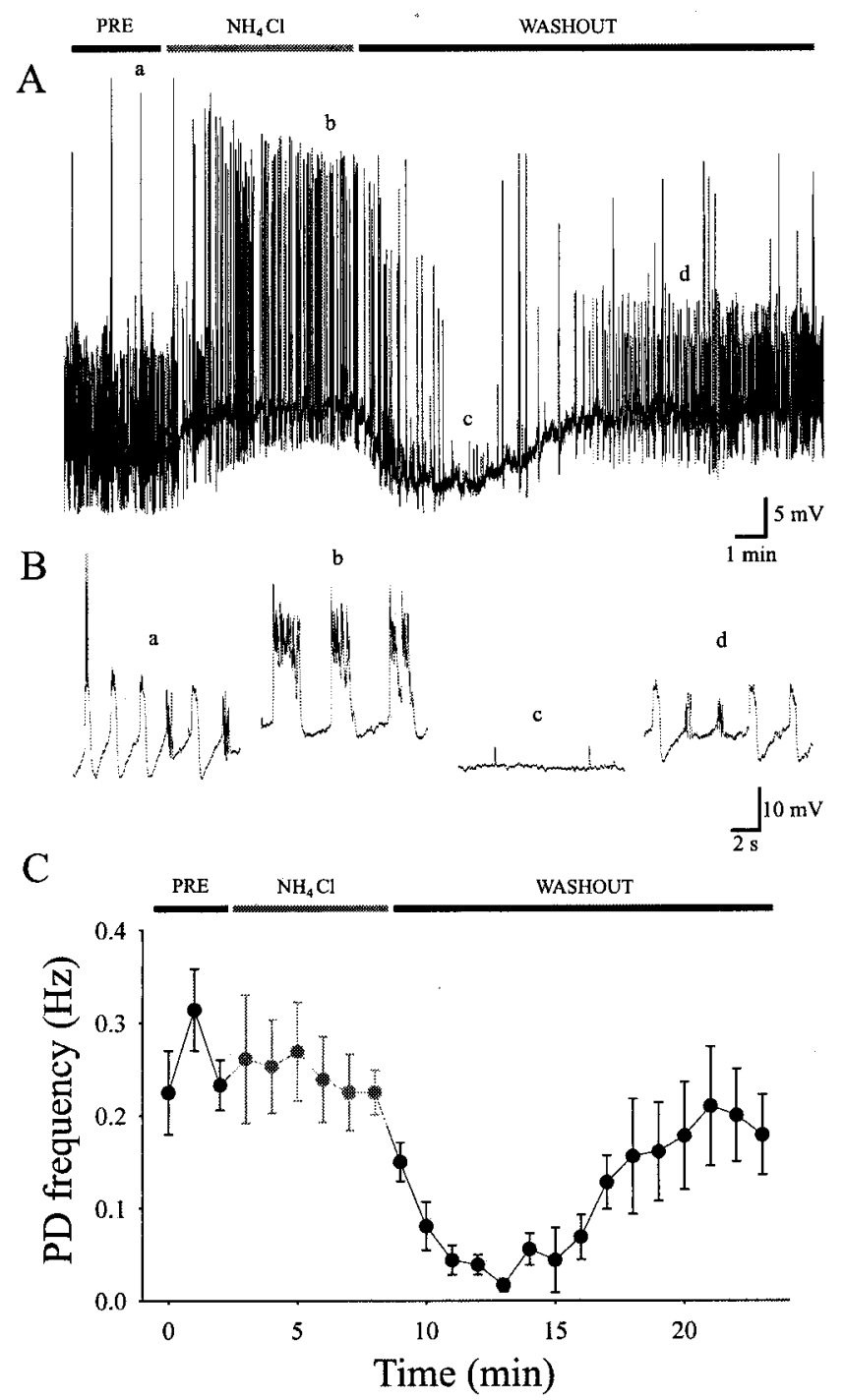

Figure 7. Effect of acidification on motoneuron activity patterns. $\mathrm{NH}_{4} \mathrm{Cl}$ was transiently applied to modify the intracellular $\mathrm{pH}$ of motoneurons. $A$ shows a current-clamp recording in which $a$ is the preapplication control, $b$ shows the application of $\mathrm{NH}_{4} \mathrm{Cl}, c$ shows the block of activity caused by the early wash-out, and $d$ is the late wash-out and return to control values. $B$ shows excerpts from $A$ on an expanded time scale. $C$, Graph showing the results for an average of six cells.

argued for a neural origin of this behavior. On the basis of observations in other preparations (Roberts 1990; Grillner et al., 1991; Bracci et al., 1996; Cazalets et al., 1996; Chub and O'Donovan 1998), we assumed that the spontaneous, alternating contractions of the zebrafish embryo would be generated by a combination of glutamatergic excitation and glycinergic inhibition. In the chick embryo, Chub and O'Donovan (1998) found that either type of blockers could transiently suppress spontaneous network activity in the spinal cord, which could be completely suppressed only in the presence of both types of blockers. Surprisingly, the spontaneous contractions and motoneuron firing patterns in the early zebrafish embryo were insensitive to either glutamate or glycine receptor blockers or both, suggesting a fundamentally different type of mechanism.

Although two types of activity were observed, periodic depolarizations seem to underlie the contractions because they were 
present at the earliest stages of development, generated action potential volleys that were in the frequency range of contractions, and as with the behavior were unaffected by blockers of glutamatergic or glycinergic receptors. Periodic inward currents had small current amplitudes $(\sim 10 \mathrm{pA})$ that produced large voltage jumps and spike volleys in current-clamp recordings resulting from the high input resistance of embryonic motoneurons. The gradual decrease in input resistance during development could explain the misalternations of the contractions and their eventual disappearance by reducing the periodic depolarizations below action potential threshold.

Because the frequency of periodic depolarizations varied with the membrane potential, this suggested that they could be generated by mechanisms intrinsic to the motoneurons. Excitatory plateau potentials recorded in the presence of TTX in adult lamprey and rats and in embryonic Xenopus (Wallen and Grillner 1987; Hochman et al., 1994; Prime et al., 1999) can be generated cell-intrinsically by an interaction between bi-stable membrane properties and activation of NMDA receptors. However, three observations indicate that this is not the case for the periodic depolarizations. First, periodic depolarizations were present at membrane voltages that spanned $-80 \mathrm{mV}$ to $-20 \mathrm{mV}$, a range likely to be too large to be subjected to bi-stable membrane properties. Second, periodic depolarizations were abolished by TTX. Third, blockade of NMDA and AMPA receptors had no effect on the generation of periodic depolarizations. Together these observations go against an intrinsic mechanism of motoneuron rhythmicity.

We next considered the role of synaptic transmission. Our inability to reverse the periodic inward currents by changing the holding potential and the lack of block by a low $\mathrm{Ca}^{2+} /$ high $\mathrm{Mg}^{2+}$ solution suggest that the presynaptic input is not chemically mediated, leaving the possibility of electrical connections. Consistent with electrical connections, we observed that heptanol and intracellular acidification (by $\mathrm{NH}_{4} \mathrm{Cl}$ rebound), known gap junction uncouplers, blocked the periodic depolarizations, and heptanol also blocked the behavior. We observed only a minute fraction of dye-coupled neurons. Given the small amplitude of the presumed junctional currents $(<10 \mathrm{pA})$ and therefore the limited number of gap junctional molecules, it would not seem unusual to have had difficulty detecting dye coupling. A similar scarcity of dye coupling has been reported for pairs of neocortical neurons showing robust electrical coupling (Gibson et al., 1999) and may be caused by the presence of dye-impermeant junctions. Electrical coupling through a small number of junctions could allow the passage of enough current to bring a high-resistance postsynaptic neuron to threshold for spike activity but would be expected to severely attenuate the passage of current during the presynaptic action potential volley. This would account for the presence of small, possibly highly filtered spikelets riding on the step currents during periodic inward currents (Fig. 3C) and for the sensitivity of the periodic inward currents to TTX.

A widespread distribution of electrical synapses has been observed by electron microscopic examination of adult mammalian spinal cord (Rash et al., 1996). An important role for electrical coupling in developing central networks has also been suggested for other vertebrates (Feller, 1999) and is a common component of invertebrate central program generators (Simmers et al., 1995). An electrical network in the early zebrafish spinal cord could explain why motoneuron depolarization increased the frequency of periodic inward currents if it reflects electrical coupling. Accordingly, depolarizing a motoneuron could lead to excitation of all coupled (e.g., ipsilateral) cells and thus an acceleration of the network oscillations. Because periodic inward currents were eliminated in TTX or $\mathrm{Cd}^{2+}$ but not $\mathrm{Co}^{2+}, \mathrm{Co}^{2+}$-insensitive calcium entry elsewhere in the premotor network appears to be essential for the pacemaker activity.

\section{Synaptic bursts and neurotransmitters}

Blockers of receptors for glutamate and acetylcholine, the ubiquitous excitatory spinal neurotransmitters, were without effect on either type of activity pattern or on the contractions. A lack of cholinergic innervation of motoneurons is not surprising because they lack collaterals that could contact other motoneurons within the spinal cord at these stages. Blocking GABA receptors did not suppress these activities but rather increased the duration of both types of events. A similar effect was observed with the low $\mathrm{Ca}^{2+} /$ high $\mathrm{Mg}^{2+}$ solution, suggesting that it may have acted by suppressing tonic GABA secretion. Tonic GABA release seems unlikely at first glance given the lack of detectable GABAergic events. Previous work on reticulospinal Mauthner neurons of the larval zebrafish hindbrain has shown the presence of abundant GABAergic terminals by immunoelectron microscopy, but only a minute fraction of all spontaneous synaptic events were GABAergic (Triller et al., 1997). These results suggest that functionally immature GABAergic synapses are present and perhaps secrete GABA in the absence of detectable quantal release, which in turn could exert a modulatory action on the spinal network. An alternative possibility is suggested by the recent observation that GABA can be co-released with glycine in the mammalian brain (Jonas et al., 1998) and spinal cord (Chéry and De Koninck, 1999), presumably because of the packaging of both transmitters in the same synaptic vesicles. If this also occurs in the zebrafish spinal cord, then perhaps very low (immature) amounts of GABA are co-released with high (mature) amounts of glycine during "glycinergic" bursts.

This brings us to consider the other main type of rhythmic activity observed in older embryos: regular bursts of glycinergic synaptic events. Synaptic bursts appeared in the majority of motoneurons at $21 \mathrm{hr}$, somewhat later than the periodic inward currents, and these newly formed synapses appeared to mature by $24 \mathrm{hr}$, as reflected by the increase in current amplitude over this $3 \mathrm{hr}$ period. Because the synaptic bursts never triggered action potentials and strychnine did not affect the behavior, glycinergic transmission is unnecessary for generating the contractions. It is therefore curious that the synaptic bursts occurred at frequencies similar to the periodic depolarizations.

We speculate that the synaptic bursts are produced by commissural interneurons projecting from the contralateral side. Commissural interneurons have been described in the early zebrafish embryo (Bernhardt et al., 1990) and play a role in midcycle inhibition during swimming episodes in Xenopus embryos and adult lamprey (Roberts 1990; Grillner et al., 1991). Reciprocal inhibition of this type could account for the comparable frequencies of both activity patterns in embryonic zebrafish motoneurons.

We have indirect evidence supporting the possibility of reciprocal inhibition. The frequency of synaptic events within each synaptic burst was highly similar to the frequency of action potentials recorded in motoneurons during periodic depolarizations. Thus between 20 and $24 \mathrm{hr}$, glycinergic event frequency increased from $11 \pm 3$ to $51 \pm 12 \mathrm{~Hz}$ and action potentials increased from $14 \pm 2$ to $43 \pm 4 \mathrm{~Hz}$; the values at each time point were not significantly different. These observations suggest that 
the glycinergic interneurons presynaptic to the motoneurons and the motoneurons themselves are firing at the same frequency and undergo a similar developmental maturation. If correct, this would indicate that a similar network excites the motoneurons and the inhibitory interneurons, and this may be the case for all active neurons of the embryonic spinal cord. Accordingly, we would expect to see an electrical component in addition to the glycinergic input from the inhibitory interneurons to the motoneurons. This appears to be the case in some recordings in which a small depolarizing step accompanied the synaptic bursts (Figs. 5B, 6D) in the presence of strychnine. This interpretation implies that the periodic depolarizations are the fundamental activity patterns that underlie both the contractions and the glycinergic synaptic bursts.

What is observed in the early embryo may be the formation of an essential component of the central program generator (CPG). This basic CPG may be modified for swimming later in the larvae when chemical inputs become predominant. Recordings from identified spinal interneurons, and in particular dual recordings with motoneurons, will be required to test this hypothesis and to define at a cellular level the neural network for spontaneous contractions.

\section{REFERENCES}

Ali DW, Buss RR, Drapeau P (2000) Properties of miniature glutamatergic EPSCs in neurones of the locomotor regions of the developing zebrafish. J Neurophysiol 83:181-191.

Bernhardt RR, Chitnis AB, Lindamer L, Kuwada JY (1990) Identification of spinal neurons in the embryonic and larval zebrafish. J Comp Neurol 302:603-616.

Bracci E, Ballerini L, Nistri A (1996) Spontaneous rhythmic bursts induced by pharmacological block of inhibition in lumbar motoneurons of the neonatal rat spinal cord. J Neurophysiol 75:640-647.

Buss RR, Ali DW, Drapeau P (1999) Properties of synaptic currents and fictive motor behaviors in neurons of the locomotor regions of the developing zebrafish. Neurosci Abstr 25:1151.

Cazalets J-R, Borde M, Clarac F (1996) The synaptic drive from the spinal locomotor network to motoneurons in the newborn rat. J Neurosci 16:298-306.

Cherubini E, Gaiarsa JL, Ben-Ari Y (1991) GABA: an excitatory transmitter in early postnatal life. Trends Neurosci 14:515-519.

Chéry N, De Koninck Y (1999) Junctional versus extrajunctional glycine and $\mathrm{GABA}_{\mathrm{A}}$ receptor-mediated IPSCs in identified lamina I neurons of the adult rat spinal cord. J Neurosci 19:7342-7355.

Chub N, O'Donovan MJ (1998) Blockade and recovery of spontaneous rhythmic activity after application of neurotransmitter antagonists to spinal networks of the chick embryo. J Neurosci 18:294-306.

Drapeau P, Ali DW, Buss RR, Saint-Amant L (1999) In vivo recording from identifiable neurons of the locomotor network in the developing zebrafish. J Neurosci Methods 88:1-13.

Evans DH (1979) Fish. In: Comparative physiology of osmoregulation in animals (Maloiy GMO, ed), pp 305-390. Orlando, FL: Academic.

Feller MB (1999) Spontaneous correlated activity in developing neural circuits. Neuron 22:653-656.

Gibson JR, Beirlein M, Connors BW (1999) Two networks of electrically coupled inhibitory neurons in neocortex. Nature 402:75-79.

Granato M, VanEeden FJM, Schach U, Trowe T, Brand M, FurutaniSeiki M, Haffter P, Hammerschmidt M, Heisenberg C-P, Jiang Y-J, Kane DA, Kelsh RN, Mullins MC, Odenthal J, Nusslein-Volhard C (1996) Genes controlling and mediating locomotion behavior of the zebrafish embryo and larvae. Development 123:399-413.

Grillner S, Wallen P, Brodin L (1991) Neuronal network generating locomotor behavior in lamprey: circuitry, transmitters, membrane properties, and stimulation. Annu Rev Neurosci 14:169-199.

Grunwald DJ, Kimmel CB, Westerfield M, Walker C, Streisinger G (1988) A neural degeneration mutation that spares primary neurons in the zebrafish. Dev Biol 126:115-128.
Hamill OP, Marty A, Neher E, Sakmann B, Sigworth FJ (1981) Improved patch clamp techniques for high-resolution current recordings from cells and cell free patches. Pflügers Arch 391:85-100.

Harris JE, Whitting HP (1954) Structure and function in the locomotory system of the dogfish embryo. The myogenic stage of movement. J Exp Biol 31:501-524.

Hochman S, Jordan LM, Schmidt BJ (1994) TTX-resistant NMDA receptor-mediated voltage oscillations in mammalian lumbar motoneurons. J Neurophysiol 72:2559-2562.

Jonas P, Bischofberger J, Sandkuhler J (1998) Corelease of two fast neurotransmitters at a central synapse. Science 281:419-424.

Katz B, Miledi R (1967) Ionic requirements of synaptic transmitter release. Nature 215:651.

Kiehn O (1991) Plateau potentials and active integration in the final common pathway for motor behavior. Trends Neurosci 14:68-73.

Kimmel CB, Ballard WW, Kimmel SR, Ullman B, Schilling TF (1995) Stages of embryonic development of the zebrafish. Dev Dyn 203:253-310.

Kuwada JY, Bernhardt RR, Nguyen N (1990) Development of spinal neurons and tracts in the zebrafish embryo. J Comp Neurol 302:617-628.

Legendre P, Korn H (1994) Glycinergic inhibitory synaptic currents and related receptor channels in the zebrafish brain. Eur $\mathrm{J}$ Neurosci 6:1544-1557.

Prime L, Pichon Y, Moore LE (1999) N-Methyl-D-aspartate-induced oscillations in whole cell clamped neurons from the isolated spinal cord of Xenopus laevis embryos. J Neurophysiol 82:1069-73.

Myers PZ, Eisen JS, Westerfield M (1986) Development and axonal outgrowth of identified motoneurons in the zebrafish. J Neurosci 6:2278-2289.

Nachshen DA, Drapeau P (1988) The regulation of cytosolic pH in isolated presynaptic nerve terminals from rat brain. J Gen Physiol 91:289-303.

Nguyen PV, Aniksztejn L, Catarsi S, Drapeau P (1999) Maturation of neuromuscular transmission during early development in zebrafish. J Neurophysiol 81:2852-2861.

Ozaki S, Yamada T, Iizuka M, Nishimaru H, Kudo N (1996) Development of locomotor activity induced by NMDA receptor activation in the lumbar spinal cord of the rat fetus studied in vitro. Dev Brain Res 97:118-125.

Rash JE, Dillman RK, Bilhartz BL, Duffy HS, Whalen LR, Yasumura T (1996) Mixed synapses discovered and mapped throughout mammalian spinal cord. Proc Natl Acad Sci USA 93:4235-4239.

Roberts A (1990) How does a nervous system produce behavior? A case study in neurobiology. Sci Prog 74:31-51.

Roos A, Boron WF (1981) Intracellular pH. Physiol Rev 61:296-434.

Saint-Amant L, Drapeau P (1998) Time course of the development of motor behaviors in the zebrafish embryo. J Neurobiol 37:622-632.

Saint-Amant L, Drapeau P (1998) Motoneuron activity patterns during spontaneous locomotor behavior of the zebrafish embryo. Soc Neurosci Abstr 654.11.

Simmers J, Meyrand P, Moulins M (1995) Modulation and dynamic specification of motor rhythm-generating circuits in crustacea. J Physiol (Paris) 89:195-208.

Singer JH, Talley EM, Bayliss DA, Berger AJ (1998) Development of glycinergic synaptic transmission to rat brain stem motoneurons. J Neurophysiol 80:2608-2620.

Spray DC, Harris AL, Bennett MVL (1981) Gap junctional conductance is a simple and sensitive function of intracellular $\mathrm{pH}$. Science 211:712-715.

Takens-Kwak BR, Jongsma HJ, Rook MB, Van Ginneken AC (1992) Mechanism of heptanol-induced uncoupling of cardiac gap junctions: a perforated patch-clamp study. Am J Physiol 262:C1531-1538.

Triller A, Rostaing P, Korn H, Legendre P (1997) Morphofunctional evidence for mature synaptic contacts on the Mauthner cell of 52-hourold zebrafish larvae. Neuroscience 80:133-145.

Wallen P, Grillner S (1987) N-Methyl-D-aspartate receptor-induced, inherent oscillatory activity in neurons active during fictive locomotion in the lamprey. J Neurosci 7:2745-2755.

Westerfield M (1995) The zebrafish book: a guide for the laboratory use of zebrafish (Brachydanio rerio). Eugene, OR: University of Oregon. 